\title{
A Complex Field Network Coding Scheme in Wireless Communication
}

\author{
Gao Xiang, Wang Liming \\ Navel University of Engineering, College of Electrical Engineering, Wuhan, China \\ E-mail: 435610133@qq.com
}

\begin{abstract}
Keywords - wireless communication, network coding, diversity gain, relay
Abstract - Complex field network coding (CFNC) is a new network coding approach proposed to improve network throughput and achieve full diversity gain in wireless networks. In this paper, a novel complex-field network coding scheme is developed based on cooperative communication. Different from traditional network coding scheme, transmissions are not restricted to binary symbols. The proposed scheme can achieve throughput to approximately XXX symbols per source per channel use. Theoretic analysis demonstrate that, the improved CFNC scheme can obtain the same network throughput and diversity gain as CFNC, while it can decrease the transmission delay of source symbols, and reduce the detection complexity of destination.
\end{abstract}

\section{Introduction}

At the turn of the century, Ahlswede and other researchers proposed the idea of network coding, by relay node to deal with information in order to improve the network capacity [1]. Before network coding technology was proposed, relay nodes only store and forward information in the process of data transmission. After scholars put forward the concept of network coding [1], the advantage of network coding is more and more studied by people, and network coding theory has been further developed. With the establishment of the theory of network coding, how to construct network coding is becoming more and more attracted to scholars. Linear combination becomes the most important means of constructing network coding scheme [3-4].

Thanks to the broadcast nature of wireless channels, capacity and diversity gains can be achieved through cooperation of distributed users. In the multicast network scenarios with only one source node, Li has further studied linear network coding. Accord to linear network coding, relay nodes combine the received information. Relay nodes treat the information as vector. The relay nodes can make linear transformation to the vectors. Li has demonstrated that if we could design perfect linear transformation algorithm, the max information flow for each link could be achieved [3]. After Li and other scholars has taken detailed research on multicast network with only one source, Koetter and some scholars explore the throughput of network coding [4]. In the case of relay nodes with decoding ability, Scholars need to further explore relay nodes for encoding and decoding complexity. Through the theoretical analysis, Jaggi demonstrated that the node could encode and decode with polynomial complexity. Ho and other scholars put forward Random Linear Network Coding. In Random Linear Network Coding, the coefficients are selected randomly [5] [6].

Network coding in communication can enhance the throughput and performance of the network. However, time delay is unavoidable owe to the complexity of encoding and decoding at the nodes. It is essential to realize network coding briefly and quickly. Too complicated way of network coding is a great challenge to relay nodes. So, linear network coding is more and more popular. The research mentioned above were discussed when network coding was just put forward. Though the early research were based on wire communication and the assumption of no delay, they laid the a solid foundation for the application of network coding in wireless communication. The research mentioned above were discussed when network coding was just put forward. Though the early research were based on wire communication and the assumption of no delay, they laid the a solid foundation for the application of network coding in wireless communication. Network coding technology has applicability in multiple fields of communications. Especially in the practical communication system, the application of network coding has many potential could be mining [3], [7]. In the research field of network coding, scholars are most interested in how to use network 
coding in wireless communication network. In the field of wireless network coding, many scholars have carried on deep discussion and research. In the literature [8] and [9], broadcast characteristics of wireless channel have been made full use of. Scholars combined broadcast characteristics with network coding, and achieved good results. In literature [10], LP equation was put forward aiming at energy saving. In literature [11], network coding was demonstrated aiming at reducing transmission delay. In literature [12], the physical layer network coding was put forward based on traditional network coding. In literature [13], considering from the perspective of network coding protocol, COPE protocol was designed. Scholars also researched the application of network coding in wireless sensor network [14][15]. In the fields of computing network throughput [17], analyzing channel characteristics [18] and reducing interference [20], network coding is also studied.

In this paper, we investigate a two-source and one-destination wireless communication system, where two source nodes transmit information to a destination node. Source nodes can also be amplify-and-forward(AF) relay nodes. The remainder of this paper is organized as follows. In Section II, the conventional complex field network coding scheme is described. In Section III, our proposed network coding scheme is described. Simulation and numerical results are provided in Section IV, and finally, our conclusions are drawn in Section V.

\section{conventional complex field network coding}

In the first time slot, source node $S_{1}$ transmits its symbols $x_{1}$ to source node $S_{2} . S_{2}$ receives the symbols from $S_{1}$ and stores the symbols, noted as $y_{12}^{1}$. At the same time, $S_{1}$ transmits its symbols $x_{1}$ to $D$. $D$ receives the symbols from $S_{1}$ and stores the symbols, noted as $y_{1 d}^{1}$. The signals received by destination node and source node $S_{2}$ are given by

$$
\begin{gathered}
y_{1 d}^{1}=h_{1 d}^{1} x_{1}+n_{1 d}^{1} \\
y_{12}^{1}=h_{12}^{1} x_{1}+n_{12}^{1}
\end{gathered}
$$

where $h_{1 d}^{1}$ and $h_{12}^{1}$ are the Rayleigh fading coefficient corresponding to the $S_{1} \rightarrow D$ and the $S_{1} \rightarrow S_{2}$ link in time slot $1 ; n_{1 d}^{1}$ and $n_{12}^{1}$ are the noise terms.

In the second time slot, $S_{2}$ transmits its symbols $x_{2}$ to $S_{1} . S_{1}$ receives the symbols from $S_{2}$ and stores the symbols. At the same time, $S_{2}$ transmits its symbols $x_{2}$ to $D . D$ receives the symbols from $S_{2}$ and stores the symbols, noted as $y_{2 d}^{2}$.

The signals received by destination node $D$ and source node $S_{2}$ are given by

$$
\begin{aligned}
& y_{2 d}^{2}=h_{2 d}^{2} x_{2}+n_{2 d}^{2} \\
& y_{21}^{2}=h_{21}^{2} x_{2}+n_{21}^{2}
\end{aligned}
$$

where $h_{2 d}^{1}$ and $h_{21}^{1}$ are the Rayleigh fading coefficient corresponding to the $S_{2} \rightarrow D$ and the $S_{2} \rightarrow S_{1}$ link in time slot $2 ; n_{2 d}^{1}$ and $n_{21}^{1}$ are the noise terms.

In the third time slot, $S_{1}$ combines the symbols received from $S_{2}$ and its own symbols by network coding, notes as $y_{1 d}^{3}$, and then transmits the signals $y_{1 d}^{3}$ to $D$.

The signals received by destination node $D$ from source node $S_{1}$ are given by

$$
y_{1 d}^{3}=h_{1 d}^{3} \frac{1}{\left|w_{11}^{3} x_{1}+w_{12}^{3} y_{21}^{2}\right|}\left(w_{11}^{3} x_{1}+w_{12}^{3} y_{21}^{2}\right)+n_{1 d}^{3}
$$

where $h_{1 d}^{3}$ is the Rayleigh fading coefficient corresponding to the $S_{1} \rightarrow D$ link in time slot $3 ; n_{1 d}^{3}$ is the noise term. $\omega_{11}^{3}$ and $\omega_{12}^{3}$ are the complex field network coding coefficient, while they are orthogonal. 
In the fourth time slot, $S_{2}$ combines the symbols received from $S_{1}$ and its own symbols by network coding, notes as $y_{2 d}^{4}$, and then transmits the signals $y_{2 d}^{4}$ to $D$.

The signals received by destination node $D$ from source node $S_{2}$ are given by

$$
y_{2 d}^{4}=h_{2 d}^{4}\left(w_{21}^{4} y_{12}^{1}+w_{22}^{4} x_{2}\right)+n_{2 d}^{4}
$$

where $h_{2 d}^{4}$ is the Rayleigh fading coefficient corresponding to the $S_{2} \rightarrow D$ link in time slot $4 ; n_{2 d}^{4}$ is the noise term. $\omega_{21}^{4}$ and $\omega_{22}^{4}$ are the complex field network coding coefficient, while they are orthogonal.

The signals that $D$ received can be given by

$$
\left[\begin{array}{l}
y_{1 d}^{1} \\
y_{2 d}^{2} \\
y_{1 d}^{3} \\
y_{2 d}^{4}
\end{array}\right]=H\left[\begin{array}{l}
x_{1} \\
x_{2}
\end{array}\right]+N
$$

Where $H$ is link matrix and $N$ is noise matrix.

At the destination node $D$, by zero-forcing algorithm, signals can be restored.

$$
\left[\begin{array}{l}
x_{1} \\
x_{2}
\end{array}\right]=H^{-1}\left[\begin{array}{c}
y_{1 d}^{1} \\
y_{2 d}^{2} \\
y_{1 d}^{3} \\
y_{2 d}^{4}
\end{array}\right]-H^{-1} N
$$

\begin{tabular}{|c|c|c|c|}
\hline Time Slot & Time Slot & Time Slot & Time Slot \\
\hline & 2 & 3 & 4 \\
\hline $\mathrm{UE} 1 \rightarrow \mathrm{x} \mathrm{YE} / \mathrm{D}$ & & $\begin{array}{l}\mathrm{UE} 1 \rightarrow \mathrm{D} \\
x_{1}+y_{21}^{2}\end{array}$ & \\
\hline & $\mathrm{UE2} \underset{\times 2}{\rightarrow} \mathrm{UE} 1 / \mathrm{D}$ & & $\begin{array}{l}\mathrm{yE} 2 \rightarrow \mathrm{D} \\
y_{12}+x_{2}\end{array}$ \\
\hline
\end{tabular}

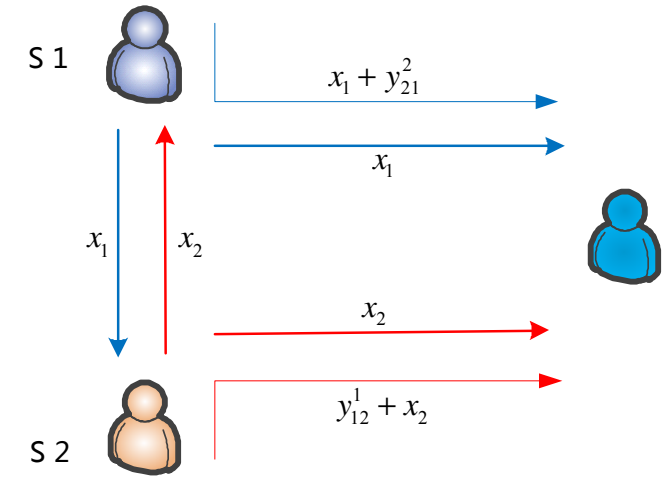

Fig.1 Conventional network coding

Fig.2 Time slots of conventional network coding

\section{proposed complex field network coding}

Proposed method of complex filed network coding is the same as the conventional one in the first two time slots. In the first time slot, $S_{1}$ transmits its symbols $x_{1}$ to $S_{2}$. $S_{2}$ receives the symbols from $S_{1}$ and stores the symbols, noted as $y_{12}^{1}$. At the same time, $S_{1}$ transmits its symbols $x_{1}$ to $D$. $D$ receives the symbols from $S_{1}$ and stores the symbols, noted as $y_{1 d}^{1}$. The signals received by destination node and source node $S_{2}$ are given by

$$
\begin{aligned}
& y_{1 d}^{1}=h_{1 d}^{1} x_{1}+n_{1 d}^{1} \\
& y_{12}^{1}=h_{12}^{1} x_{1}+n_{12}^{1}
\end{aligned}
$$


where $h_{1 d}^{1}$ and $h_{12}^{1}$ are the Rayleigh fading coefficient corresponding to the $S_{1} \rightarrow D$ and the $S_{1} \rightarrow$ $S_{2}$ link in time slot $1 ; n_{1 d}^{1}$ and $n_{12}^{1}$ are the noise terms.

In the second time slot, $S_{2}$ transmits its symbols $x_{2}$ to $S_{1} . S_{1}$ receives the symbols from $S_{2}$ and stores the symbols. At the same time, $S_{2}$ transmits its symbols $x_{2}$ to $D$. $D$ receives the symbols from $S_{2}$ and stores the symbols, noted as $y_{2 d}^{2}$.

The signals received by destination node ${ }_{\mathrm{V}}$ and source node $S_{2}$ are given by

$$
\begin{aligned}
& y_{2 d}^{2}=h_{2 d}^{2} x_{2}+n_{2 d}^{2} \\
& y_{21}^{2}=h_{21}^{2} x_{2}+n_{21}^{2}
\end{aligned}
$$

where $h_{2 d}^{1}$ and $h_{21}^{1}$ are the Rayleigh fading coefficient corresponding to the $S_{2} \rightarrow D$ and the $S_{2} \rightarrow$ $S_{1}$ link in time slot $2 ; n_{2 d}^{1}$ and $n_{21}^{1}$ are the noise terms.

In the third time slot, $S_{1}$ combines the symbols received from $S_{2}$ and its own symbols by complex field network coding, notes as $y_{1 d}^{3}$, and then transmits the signals $y_{1 d}^{3}$ to $D$. At the same time, $S_{2}$ combines the symbols received from $S_{1}$ and its own symbols by complex field network coding, notes as $y_{2 d}^{3}$, and then transmits the signals $y_{2 d}^{3}$ to $D$.

The signals received by destination node $D$ from source node $S_{1}$ and source node $S_{2}$ are given by

$$
\begin{aligned}
& y_{1 d}^{3}=h_{1 d}^{3} \frac{1}{\left|w_{11}^{3} x_{1}+w_{12}^{3} y_{21}^{2}\right|}\left(w_{11}^{3} x_{1}+w_{12}^{3} y_{21}^{2}\right)+n_{1 d}^{3} \\
& y_{2 d}^{3}=h_{2 d}^{3} \frac{1}{\left|w_{21}^{3} y_{12}^{1}+w_{22}^{3} x_{2}\right|}\left(w_{21}^{3} y_{12}^{1}+w_{22}^{3} x_{2}\right)+n_{2 d}^{3}
\end{aligned}
$$

where $h_{1 d}^{3}$ and $h_{2 d}^{3}$ are the Rayleigh fading coefficient corresponding to the $S_{1} \rightarrow D$ and $S_{2} \rightarrow D$ link in time slot $3 ; n_{1 d}^{3}$ and $n_{2 d}^{3}$ are the noise terms. $\omega_{11}^{3}, \omega_{12}^{3}, \omega_{21}^{3}$ and $\omega_{21}^{3}$ are the complex field network coding coefficient, while they are orthogonal.

In the fourth time slot, $S_{2}$ combines the symbols received from $S_{1}$ and its own symbols by network coding, notes as $y_{1 d}^{4}$, and then transmits the signals $y_{1 d}^{4}$ to $D$. At the same time, $S_{1}$ combines the symbols received from $S_{2}$ and its own symbols by complex field network coding, notes as $y_{2 d}^{4}$, and then transmits the signals $y_{2 d}^{4}$ to $D$.

The signals received by destination node from source node $S_{1}$ and source node $S_{2}$ are given by

$$
\begin{array}{r}
y_{1 d}^{4}=h_{1 d}^{4} \frac{1}{\left|w_{11}^{4} x_{1}+w_{12}^{4} y_{21}^{2}\right|}\left(w_{11}^{4} x_{1}+w_{12}^{4} y_{21}^{2}\right)+n_{1 d}^{4} \\
y_{2 d}^{4}=h_{2 d}^{4} \frac{1}{\left|w_{21}^{4} y_{12}^{1}+w_{22}^{4} x_{2}\right|}\left(w_{21}^{4} y_{12}^{1}+w_{22}^{4} x_{2}\right)+n_{2 d}^{4}
\end{array}
$$

where $h_{1 d}^{4}$ and $h_{2 d}^{4}$ are the Rayleigh fading coefficient corresponding to the $S_{1} \rightarrow D$ and $S_{2} \rightarrow D$ link in time slot $4 ; n_{1 d}^{4}$ and $n_{2 d}^{4}$ are the noise terms. $\omega_{11}^{4}, \omega_{12}^{4}, \omega_{21}^{4}$ and $\omega_{21}^{4}$ are the complex field network coding coefficient, while they are orthogonal.

The signals that $D$ received can be given by

$$
\left[\begin{array}{c}
y_{1 d}^{1} \\
y_{2 d}^{2} \\
y_{1 d}^{3}+y_{2 d}^{3} \\
y_{2 d}^{4}+y_{2 d}^{4}
\end{array}\right]=H\left[\begin{array}{l}
x_{1} \\
x_{2}
\end{array}\right]+N
$$

While $H$ is link matrix and $N$ is noise matrix . At the destination node $D$, by zero-forcing algorithm, signals can be restored. 


$$
\left[\begin{array}{c}
x_{1} \\
x_{2}
\end{array}\right]=H^{-1}\left[\begin{array}{c}
y_{1 d}^{1} \\
y_{2 d}^{2} \\
y_{1 d}^{3}+y_{1 d}^{3} \\
y_{2 d}^{4}+y_{2 d}^{4}
\end{array}\right]-H^{-1} N
$$

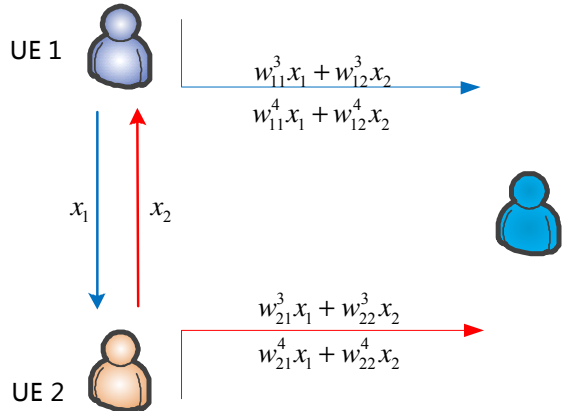

Fig.3 Proposed network coding

\begin{tabular}{|c|c|c|c|}
\hline Time Slot & \multirow{3}{*}{$\begin{array}{c}\text { Time Slot } \\
2\end{array}$} & Time Slot & \multirow{3}{*}{$\begin{array}{c}\text { Time Slot } \\
4 \\
w_{11}^{4} x_{1}+w_{12}^{4} x_{2} \\
w_{21}^{4} x_{1}+w_{22}^{4} x_{2}\end{array}$} \\
\hline 1 & & 3 & \\
\hline UE $1 \rightarrow \underset{x 1}{ }$ UE2 & & & \\
\hline & UE $\underset{\times 2}{\rightarrow}$ UE1 & $w_{21}^{3} x_{1}+w_{22}^{3} x$ & $\mathrm{UE} 2 \rightarrow \mathrm{D}$ \\
\hline
\end{tabular}

Fig.4 Time slots of proposed network coding

\section{Simulation Results And Analysis}

By conventional complex field network coding, 2 sources send 2 symbols to destination in 4 time slots. The throughput is $1 / 4 \mathrm{sym} \cdot \mathrm{S}^{-1} \cdot T S^{-1}$. The space diversity gain is 3 .

By the proposed complex field network coding, 2 sources also send 2 symbols to destination in 4 time slots. The throughput is $1 / 4 \mathrm{sym} \cdot S^{-1} \cdot T S^{-1}$. The space diversity gain is 5 .

The simulation result is shown as Fig.5. From the simulation result, we can obtain the follow observation: The outage probability of proposed network coding scheme is lower than conventional scheme under different SNR.

TABLE 1 Throughput and diversity gain of two schemes

\begin{tabular}{|c|c|c|}
\hline & Throughput & Diversity Gain \\
\hline Conventioanl & $1 / 4 \mathrm{sym} \cdot \mathrm{S}^{-1} \cdot T S^{-1}$ & 3 \\
\hline Proposed & $1 / 4 \mathrm{sym} \cdot \mathrm{S}^{-1} \cdot T S^{-1}$ & 5 \\
\hline
\end{tabular}

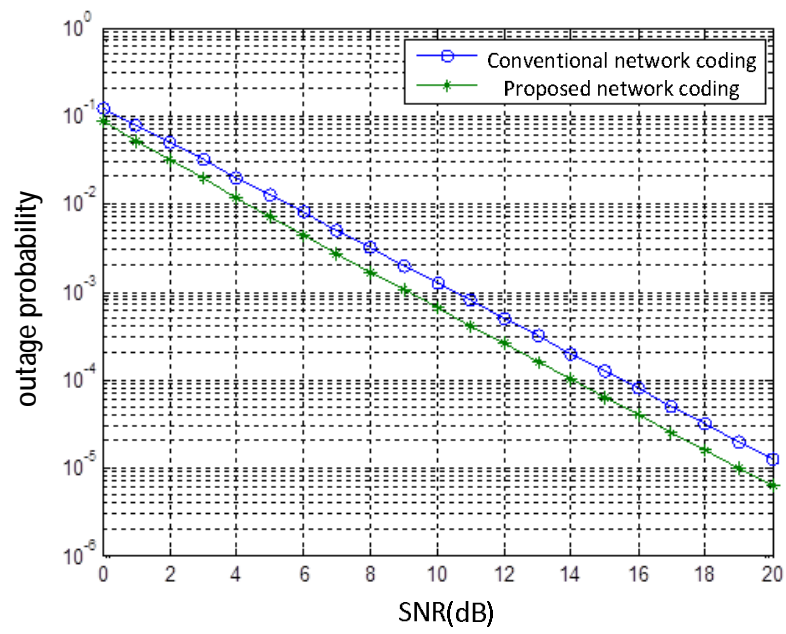

Fig.5 Outage Probability of two schemes 


\section{Conclusion}

In this paper, we have proposed a new network coding scheme. Proposed complex field network coding could prompt the performance of network because more signals are transmitted in the third and fourth time slot. The diversity gain could be enhanced. The outage probability of proposed network coding scheme is also lower than conventional scheme. in the third and fourth time slot. The diversity gain could be enhanced. The outage probability of proposed network coding scheme is also lower than conventional scheme.

However, signal processing at the nodes is becoming more complex. Network delay is likely to increase as the complexity of the terminal. The terminal will become more inefficient.

\section{References}

[1] R. W. Yeung and Z. Zhang. Distributed source coding for satellite communications. IEEE Transactions on Information Theory, vol. IT-45, pp. 1111-1120, 1999.

[2] R. Ahlswede, N. Cai, S.-Y. R. Li, and R. W. Yeung. Network information flow. IEEE Transactions on Information Theory, vol. IT-46, pp. 1204-1216, 2000.

[3] S. Y. R. Li, R. W. Yeung, and N. Cai. Linear network coding. IEEE Transactions on Information Theory, vol. 49, pp. 371-381, Feb 2003.

[4] R. Koetter and M. Medard. An algebraic approach to network coding. IEEE-ACM Transactions on Networking, vol. 11, pp. 782-795, Oct 2003.

[5] S. Jaggi, P. Sanders, P. A. Chou, M. Effros, S. Egner, K. Jain, and L. M. G. A. Tolhuizen. Polynomial time algorithms for multicast network code construction. IEEE Transactions on Information Theory, vol. 51, pp. 1973-1982, Jun 2005.

[6] T. Ho, M. Medard, R. Koetter, D. R. Karger, M. Effros, J. Shi, and B. Leong, "A random linear network coding approach to multicast," Ieee Transactions on Information Theory, vol. 52, pp. 4413-4430, Oct 2006.

[7] C. Fragouli and E. Soljanin. Network Coding Fundamentals. Foundations and Trends in Networking. vol. 1: now Publishers Inc., 2007, pp. 1-133.

[8] Y. N. Wu, P. A. Chou, and S. Y. Kung. Minimum-energy multicast in mobile ad hoc networks using network coding. IEEE Transactions on Communications, vol. 53, pp. 1906-1918, Nov 2005.

[9] C. Fragouli, J. Widmer, and J. Y. Le Boudec. A network coding approach to energy efficient broadcasting: from theory to practice. 25th IEEE International Conference on Computer Communications, Vols 1-7, Proceedings Ieee Infocom 2006, pp. 75-85.

[10]D. S. Lun. Efficient operation od coded packet networks. PhD thesis, Massachusetts Institute of Technology, June 2006, 2006.

[11]A. Eryilmaz, A. Ozdaglar, and M. Medard. On delay performance gains from network coding. 2006 40th Annual Conference on Information Sciences and Systems, Vols 1-4, pp. 864-870, 2006.

[12] S. Zhang, S. Liew, and P. Lam. Physical layer network coding. in ACM MobiCom 2006, 2006, pp. 24-29.

[13] S. Katti, H. Rahul, W. Hu, D. Katabi, M. Medard, and J. Crowcroft. XORs in the air: Practical wireless network coding. Computer Communication Review, vol. 36, pp. 243-254, Oct 2006.

[14]A. G. Dimakis, V. Prabhakaran, and K. Ramchandran. Ubiquitous access to distributed data in large-scale networks through decentralized erasure codes. Symposium on Information Processing in Sensor Networks (IPSN'05), April 2005, 2005.

[15]C. Fragouli, J. Widmer, and J. Y. Le Boudec. On the benefits of network coding for wireless applications. 2006 4th International Symposium on Modeling and Optimization in Mobile, Ad Hoc and Wireless Networks, Vols 1 and 2, pp. 679-684, 2006. 
[16]D. Petrovic, K. Ramchandran, and J. Rabaey. Overcoming untuned radios in wireless networks with network coding. IEEE Transactions on Information Theory, vol. 52, pp. 2649-2657, Jun 2006.

[17]R. Gowaikar, A. F. Dana, and J.-Y. L. Boudec. On the capacity of wireless erasure networks. Proceeding of the 2007 IEEE International Symposium on Information Theory, p. 401, 2004 